\title{
Numerical approximation of time-fractional Burgers-type equation
}

Miaomiao Yang ${ }^{1 *}$

\section{"Correspondence:}

mathxinxi@163.com

${ }^{1}$ General Education Center,

Zhengzhou Business University,

Zhengzhou, P.R. China sharing, adaptation, distribution and reproduction in any medium or format, as long as you give appropriate credit to the original author(s) and the source, provide a link to the Creative Commons licence, and indicate if changes were made. The images or other third party material in this article are included in the article's Creative Commons licence, unless indicated otherwise in a credit line to the material. If material is not included in the article's Creative Commons licence and your intended use is not permitted by statutory regulation or exceeds the permitted use, you will need to obtain permission directly from the copyright holder. To view a copy of this licence, visit http://creativecommons.org/licenses/by/4.0/.

\section{Introduction}

Burgers equation, which is used to model turbulent fluid motion, was first introduced by Harry Bateman and later studied by Burgers [1] in 1948. It plays an important role in various areas of applied mathematics, such as traffic flow, fluid mechanics, and nonlinear acoustics. Fractional calculus is a branch of mathematical analysis that studies several different possibilities of defining real number powers or complex number powers of the differentiation operator. Fractional differential equations, also known as extraordinary differential equations, are a generalization of differential equations through the application of fractional calculus. In the past decades, fractional differential equations have been applied in many fields, and, in fact, some physical processes can be modeled more accurately by using fractional derivatives [4, 7-12, 19, 21]. Recently, fractional Burgers-type equation was introduced and studied since the adding effect of the wall friction through the boundary layer can be modeled by fractional derivatives.

Some methods have been introduced to solve fractional Burgers-type equation. Vong and Lyu [13] studied a linearized finite difference scheme for a time-fractional Burgerstype equation and proved that the scheme was unconditionally convergent with second order in maximum-norm. In [3], parametric spline functions were used to solve the timefractional Burgers equation. Wang [14] studied the Adomian decomposition method for the equation. In [5], Inc presented a variational iteration method for space- and timefractional Burgers equations and obtained some exact and approximate solutions. 
In this paper, we consider the following time-fractional Burgers-type equation:

$$
\begin{aligned}
& \frac{\partial^{\alpha} u(x, t)}{\partial t^{\alpha}}+u u_{x}-\lambda_{1} u_{x x}=f(x, t), \quad(x, t) \in[a, b] \times[0, T], \\
& u(x, 0)=u_{0}(x), \quad x \in[a, b],
\end{aligned}
$$

where $0<\alpha<1$ is the order of the fractional derivatives, $f, u_{0}$ are given smooth functions, $\lambda_{1} \geq 0$ is a real parameter. The solution is considered to be either periodic or compactly supported in this paper.

The Caputo fractional derivative $\frac{\partial^{\alpha} u(x, t)}{\partial t^{\alpha}}$ is defined as follows:

$$
\frac{\partial^{\alpha} u(x, t)}{\partial t^{\alpha}}=\frac{1}{\Gamma(1-\alpha)} \int_{0}^{t} \frac{\partial u(x, s)}{\partial s} \frac{d s}{(t-s)^{\alpha}}, \quad 0<\alpha<1,
$$

where $\Gamma(\cdot)$ is the gamma function.

The paper is organized as follows. In Sect. 2, some notations and auxiliary results are described. In Sect. 3, we present the local discontinuous Galerkin method for fractional equation (1.1) and prove that the scheme is unconditionally stable and the numerical solution is convergent. Some numerical examples, given in Sect. 4, are presented to illustrate the accuracy of the method, and concluding remarks are provided in Sect. 5.

\section{Notations and auxiliary results}

In this section we introduce some notations, projections, and the numerical flux.

\subsection{Notations and projections}

Let $a=x_{\frac{1}{2}}<x_{\frac{3}{2}}<\cdots<x_{N+\frac{1}{2}}=b$ be a partition of $\Omega=[a, b]$, denote $I_{j}=\left[x_{j-\frac{1}{2}}, x_{j+\frac{1}{2}}\right]$ for $j=1, \ldots, N$, and $h_{j}=x_{j+\frac{1}{2}}-x_{j-\frac{1}{2}}, 1 \leq j \leq N, h=\max _{1 \leq j \leq N} h_{j}$.

Let us denote $u_{j+\frac{1}{2}}^{+}=\lim _{t \rightarrow 0^{+}} u\left(x_{j+\frac{1}{2}}+t\right), u_{j+\frac{1}{2}}^{-}=\lim _{t \rightarrow 0^{+}} u\left(x_{j+\frac{1}{2}}-t\right)$, and $[u]_{j+\frac{1}{2}}=$ $u_{j+\frac{1}{2}}^{+}-u_{j+\frac{1}{2}}^{+}$.

Denote by $V_{h}^{k}$ the space of piecewise discontinuous polynomials of the degree up to $k$ :

$$
V_{h}^{k}=\left\{v: v \in P^{k}\left(I_{j}\right), x \in I_{j}, j=1,2, \ldots, N\right\} .
$$

The following two projections in one dimension $[a, b]$ will be used when proving error estimate: projection $\mathcal{P}$

$$
\int_{I_{j}}(\mathcal{P} \omega(x)-\omega(x)) v(x)=0, \quad \forall v \in P^{k}\left(I_{j}\right)
$$

and projection $\mathcal{P}^{ \pm}$

$$
\begin{aligned}
& \int_{I_{j}}\left(\mathcal{P}^{+} \omega(x)-\omega(x)\right) v(x)=0, \quad \forall v \in P^{k-1}\left(I_{j}\right), \\
& \mathcal{P}^{+} \omega\left(x_{j-\frac{1}{2}}^{+}\right)=\omega\left(x_{j-\frac{1}{2}}\right),
\end{aligned}
$$


and

$$
\begin{aligned}
& \int_{I_{j}}\left(\mathcal{P}^{-} \omega(x)-\omega(x)\right) v(x)=0, \quad \forall v \in P^{k-1}\left(I_{j}\right), \\
& \mathcal{P}^{-} \omega\left(x_{j+\frac{1}{2}}^{-}\right)=\omega\left(x_{j+\frac{1}{2}}\right) .
\end{aligned}
$$

The approximation results for the projections $\mathcal{P}$ and $\mathcal{P}^{ \pm}$hold $[2,15,18,20]$

$$
\|\vartheta\|+h\|\vartheta\|_{\infty}+h^{\frac{1}{2}}\|\vartheta\|_{\tau_{h}} \leq C h^{k+1},
$$

where $\vartheta=\mathcal{P} \omega-\omega$ or $\vartheta=\mathcal{P}^{ \pm} \omega-\omega$. The positive constant $C$, solely depending on $\omega$, is independent of $h$. Here $\tau_{h}$ is the union of all element interface points, and the $L^{2}$-norm on $\tau_{h}$ is defined by

$$
\|\vartheta\|_{\tau_{h}}=\left[\sum_{1 \leq j \leq N}\left(\left(\vartheta_{j+\frac{1}{2}}^{+}\right)^{2}+\left(\vartheta_{j+\frac{1}{2}}^{-}\right)^{2}\right)\right]^{\frac{1}{2}} .
$$

In the present paper the usual notation of norms in Sobolev spaces is used. Let the scalar inner product on $L^{2}(\Omega)$ be denoted by $(\cdot, \cdot)_{\Omega}$, and the associated norm by $\|\cdot\|_{\Omega}$.

\subsection{A quantity related to the numerical flux}

$\widehat{g}\left(w^{-}, w^{+}\right)$is a given monotone numerical flux which is related to the discontinuous Galerkin discretization in space. It depends on the two values of the function $g$ at the point $x_{j+\frac{1}{2}}$, that is, $w_{j+\frac{1}{2}}^{ \pm}=w\left(x_{j+\frac{1}{2}}^{ \pm}\right)$, and satisfies:

(i) locally Lipschitz continuous;

(ii) consistent with the flux $g(w)$, that is, $\widehat{g}(w, w)=g(w)$;

(iii) a nondecreasing function of its first argument and a nonincreasing function of its second argument.

\section{Fully discrete LDG scheme}

Set $\Delta t=T / M$ to be the time mesh size, $M$ is a positive integer, $t_{j}=j \Delta t, j=0,1, \ldots, M$, is a mesh point. First we estimate the time-fractional derivative $\frac{\partial^{\alpha} u(x, t)}{\partial t^{\alpha}}$ at $t_{n}$ as follows [6, 17]:

$$
\frac{\partial^{\alpha} u\left(x, t_{n}\right)}{\partial t^{\alpha}}=\frac{(\Delta t)^{1-\alpha}}{\Gamma(2-\alpha)} \sum_{i=0}^{n-1} s_{i} \frac{u\left(x, t_{n-i}\right)-u\left(x, t_{n-i-1}\right)}{\Delta t}+\gamma^{n}(x)
$$

where $s_{i}=(i+1)^{1-\alpha}-i^{1-\alpha}, \gamma^{n}(x)$ is the truncation error

$$
\begin{aligned}
\gamma^{n}(x)= & \frac{1}{\Gamma(1-\alpha)} \sum_{i=0}^{n} \int_{t_{i-1}}^{t_{i}} \frac{\partial u(x, s)}{\partial s} \frac{d s}{\left(t_{n}-s\right)^{\alpha}}-\frac{1}{\Gamma(1-\alpha)} \sum_{i=0}^{n} \frac{\partial u\left(x, t_{i-\frac{1}{2}}\right)}{\partial t} \int_{t_{i-1}}^{t_{i}} \frac{d s}{\left(t_{n}-s\right)^{\alpha}} \\
& +\frac{(\Delta t)^{1-\alpha}}{\Gamma(2-\alpha)} \sum_{i=0}^{n-1} s_{i}\left(\frac{\partial}{\partial t} u\left(x, t_{n-i-\frac{1}{2}}\right)-\frac{u\left(x, t_{n-i}\right)-u\left(x, t_{n-i-1}\right)}{\Delta t}\right) .
\end{aligned}
$$

By some analysis, we know [16]

$$
\left\|\gamma^{n}(x)\right\| \leq C(\Delta t)^{2-\alpha}
$$

where $C$ is a constant depending on $u, T, \alpha$. 
Rewrite Eq. (1.1) as a first-order system:

$$
p=u_{x}, \quad \frac{\partial^{\alpha} u(x, t)}{\partial t^{\alpha}}+g(u)_{x}-\lambda_{1} p_{x}=f
$$

where $g(u)=\frac{1}{2} u^{2}$.

Let $u_{h}^{n}, p_{h}^{n} \in V_{h}^{k}$ be the approximation of $u\left(\cdot, t_{n}\right), p\left(\cdot, t_{n}\right)$, respectively, $f^{n}(x)=f\left(x, t_{n}\right)$. Then a fully discrete local discontinuous Galerkin method is given: find $u_{h}^{n}, p_{h}^{n} \in V_{h}^{k}$ such that, for all test functions $v, w \in V_{h}^{k}$,

$$
\begin{gathered}
\int_{\Omega} u_{h}^{n} v d x-\chi\left(\int_{\Omega} g\left(u_{h}^{n}\right) v_{x} d x-\sum_{j=1}^{N}\left(\left(\widehat{g} v^{-}\right)_{j+\frac{1}{2}}-\left(\widehat{g} v^{+}\right)_{j-\frac{1}{2}}\right)\right) \\
\quad+\chi \lambda_{1}\left(\int_{\Omega} p_{h}^{n} v_{x} d x-\sum_{j=1}^{N}\left(\left(\widehat{p_{h}^{n}} v^{-}\right)_{j+\frac{1}{2}}-\left(\widehat{p_{h}^{n}} v^{+}\right)_{j-\frac{1}{2}}\right)\right) \\
=\sum_{i=1}^{n-1}\left(s_{i-1}-s_{i}\right) \int_{\Omega} u_{h}^{n-i} v d x+s_{n-1} \int_{\Omega} u_{h}^{0} v d x+\chi \int_{\Omega} f^{n} v d x, \\
\int_{\Omega} p_{h}^{n} w d x+\int_{\Omega} u_{h}^{n} w_{x} d x-\sum_{j=1}^{N}\left(\left(\widehat{u_{h}^{n}} w^{-}\right)_{j+\frac{1}{2}}-\left(\widehat{u_{h}^{n}} w^{+}\right)_{j-\frac{1}{2}}\right)=0,
\end{gathered}
$$

where $\chi=(\Delta t)^{\alpha} \Gamma(2-\alpha)$.

The flux $\widehat{g}\left(\left(u_{h}^{n}\right)^{-},\left(u_{h}^{n}\right)^{+}\right)$is a monotone flux as described in (2.2). In order to simplify the computation, we can choose the flux

$$
\begin{aligned}
& \widehat{g}^{\mathrm{LF}}\left(w^{-}, w^{+}\right)=\frac{1}{2}\left(g\left(w^{-}\right)+g\left(w^{+}\right)-\lambda_{0}\left(w^{+}-w^{-}\right)\right), \quad \lambda_{0}=\max _{w}\left|g^{\prime}(w)\right|, \\
& \widehat{u_{h}^{n}}=\left(u_{h}^{n}\right)^{-}, \quad \widehat{p_{h}^{n}}=\left(p_{h}^{n}\right)^{+} .
\end{aligned}
$$

Remark that the crucial part for flux (3.6) is taking $\widehat{u_{h}^{n}}$ and $\widehat{p_{h}^{n}}$ from opposite sides.

Because the problem is nonlinear, an iterative method should be used when computing: find $u_{h}^{n, m}, p_{h}^{n, m} \in V_{h}^{k}$ such that, for all test functions $v, w \in V_{h}^{k}$,

$$
\begin{aligned}
& \int_{\Omega} u_{h}^{n, m} v d x+\chi \lambda_{1}\left(\int_{\Omega} p_{h}^{n, m} v_{x} d x-\sum_{j=1}^{N}\left(\left(\widehat{p_{h}^{n, m}} v^{-}\right)_{j+\frac{1}{2}}-\left(\widehat{p_{h}^{n, m}} v^{+}\right)_{j-\frac{1}{2}}\right)\right) \\
& =\sum_{i=1}^{n-1}\left(s_{i-1}-s_{i}\right) \int_{\Omega} u_{h}^{n-i} v d x+s_{n-1} \int_{\Omega} u_{h}^{0} v d x+\chi \int_{\Omega} f^{n} v d x \\
& \left.\quad+\chi\left(\int_{\Omega} g\left(u_{h}^{n, m-1}\right) v_{x} d x-\sum_{j=1}^{N}\left(\left(g\left(\widehat{u_{h}^{n, m-1}}\right) v^{-}\right)_{j+\frac{1}{2}}-\left(g \widehat{\left(u_{h}^{n, m-1}\right.}\right) v^{+}\right)_{j-\frac{1}{2}}\right)\right), \\
& \int_{\Omega} p_{h}^{n, m} w d x+\int_{\Omega} u_{h}^{n, m} w_{x} d x-\sum_{j=1}^{N}\left(\left(\widehat{u_{h}^{n, m}} w^{-}\right)_{j+\frac{1}{2}}-\left(\widehat{u_{h}^{n, m}} w^{+}\right)_{j-\frac{1}{2}}\right)=0,
\end{aligned}
$$

where $m$ is the iterative step.

Without loss of generality, we consider the case $f=0$ in numerical analysis. For the stability of scheme (3.5), we have the following result. 
Theorem 3.1 Suppose that $u_{h}^{n}$ is the solution of numerical scheme (3.5), if the numerical flux (3.6) is used, it will hold that

$$
\left\|u_{h}^{n}\right\|_{\Omega}^{2}+\chi \lambda_{1}\left\|p_{h}^{n}\right\|_{\Omega}^{2} \leq\left\|u_{h}^{0}\right\|_{\Omega}^{2}, \quad n=0,1, \ldots, M
$$

Proof We prove Theorem 3.1 by mathematical induction. First, for notational convenience, we introduce the following notations:

$$
\begin{aligned}
\widetilde{G}\left(u_{h}^{n}\right)=- & \left(\int_{\Omega} g\left(u_{h}^{n}\right)\left(u_{h}^{n}\right)_{x} d x-\sum_{j=1}^{N}\left(\left(\widehat{g}\left(u_{h}^{n}\right)^{-}\right)_{j+\frac{1}{2}}-\left(\widehat{g}\left(u_{h}^{n}\right)^{+}\right)_{j-\frac{1}{2}}\right)\right), \\
\Lambda\left(p_{h}^{n}, q_{h}^{n}, u_{h}^{n}\right)= & \left(p_{h}^{n}\right)^{-}\left(u_{h}^{n}\right)^{-}-\left(p_{h}^{n}\right)^{+}\left(u_{h}^{n}\right)^{-}-\left(u_{h}^{n}\right)^{-}\left(p_{h}^{n}\right)^{-}, \\
\Upsilon\left(p_{h}^{n}, q_{h}^{n}, u_{h}^{n}\right)= & \left(p_{h}^{n}\right)^{-}\left(u_{h}^{n}\right)^{-}-\left(p_{h}^{n}\right)^{+}\left(u_{h}^{n}\right)^{+}-\left(p_{h}^{n}\right)^{+}\left(u_{h}^{n}\right)^{-}+\left(p_{h}^{n}\right)^{+}\left(u_{h}^{n}\right)^{+} \\
& -\left(u_{h}^{n}\right)^{-}\left(p_{h}^{n}\right)^{-}+\left(u_{h}^{n}\right)^{-}\left(p_{h}^{n}\right)^{+} .
\end{aligned}
$$

We consider the case $n=1$ in (3.5) and take the test functions $v=u_{h}^{1}, w=\chi \lambda_{1} p_{h}^{1}$, then we have

$$
\begin{aligned}
\left\|u_{h}^{1}\right\|_{\Omega}^{2} & +\chi \lambda_{1}\left\|p_{h}^{1}\right\|_{\Omega}^{2}+\chi \widetilde{G}\left(u_{h}^{1}\right)+\sum_{j=1}^{N} \chi \lambda_{1}\left(\Lambda\left(p_{h}^{1}, q_{h}^{1}, u_{h}^{1}\right)_{j+\frac{1}{2}}\right. \\
& \left.-\Lambda\left(p_{h}^{1}, q_{h}^{1}, u_{h}^{1}\right)_{j-\frac{1}{2}}+\Upsilon\left(p_{h}^{1}, q_{h}^{1}, u_{h}^{1}\right)_{j-\frac{1}{2}}\right) \\
\leq & \frac{1}{2}\left\|u_{h}^{1}\right\|_{\Omega}^{2}+\frac{1}{2}\left\|u_{h}^{0}\right\|_{\Omega}^{2} .
\end{aligned}
$$

Next we deal with the term $\widetilde{G}\left(u_{h}^{1}\right)$. We define

$$
F(u)=\int^{u} g(u) d u,
$$

then

$$
\int_{\Omega} g\left(u_{h}^{1}\right)\left(u_{h}^{1}\right)_{x} d x=\sum_{j=1}^{N}\left(F\left(\left(u_{h}^{1}\right)_{j+\frac{1}{2}}^{-}\right)-F\left(\left(u_{h}^{1}\right)_{j-\frac{1}{2}}^{+}\right)\right) .
$$

Based on the monotonicity of flux $\widehat{g}$ and the mean value theorem, we have

$$
\widetilde{G}\left(u_{h}^{1}\right)=\left(F^{\prime}(\eta)-\widehat{g}\right)\left[u_{h}^{1}\right] \geq 0 .
$$

By some manual calculation, we have

$$
\Upsilon\left(p_{h}^{1}, q_{h}^{1}, u_{h}^{1}\right)_{j-\frac{1}{2}}=0,
$$

that is,

$$
\left\|u_{h}^{1}\right\|_{\Omega}^{2}+\chi \lambda_{1}\left\|p_{h}^{1}\right\|_{\Omega}^{2} \leq\left\|u_{h}^{0}\right\|_{\Omega}^{2}
$$


and

$$
\left\|u_{h}^{1}\right\|_{\Omega} \leq\left\|u_{h}^{0}\right\|_{\Omega} .
$$

Suppose that

$$
\left\|u_{h}^{k}\right\|_{\Omega} \leq\left\|u_{h}^{0}\right\|_{\Omega}, \quad k=1,2, \ldots, P
$$

Considering the case $n=P+1$ in scheme (3.5) and taking the test functions $v=u_{h}^{P+1}$, $\omega=\chi \lambda_{1} p_{h}^{P+1}$, we have

$$
\begin{gathered}
\int_{\Omega}\left(u_{h}^{P+1}\right)^{2} d x+\chi \widetilde{G}\left(u_{h}^{P+1}\right)+\sum_{j=1}^{N} \chi \lambda_{1}\left(\Lambda\left(p_{h}^{P+1}, q_{h}^{P+1}, u_{h}^{P+1}\right)_{j+\frac{1}{2}}\right. \\
\left.-\Lambda\left(p_{h}^{P+1}, q_{h}^{P+1}, u_{h}^{P+1}\right)_{j-\frac{1}{2}}+\Upsilon\left(p_{h}^{P+1}, q_{h}^{P+1}, u_{h}^{P+1}\right)_{j-\frac{1}{2}}\right) \\
\leq\left(\sum_{i=1}^{P}\left(s_{i-1}-s_{i}\right)+s_{P}\right)\left\|u_{h}^{0}\right\|_{\Omega}\left\|u_{h}^{P+1}\right\|_{\Omega} .
\end{gathered}
$$

Analogous to the proofs of inequalities (3.11) and (3.12), we know

$$
\widetilde{G}\left(u_{h}^{P+1}\right) \geq 0, \quad \Upsilon_{j-\frac{1}{2}}\left(p_{h}^{P+1}, q_{h}^{P+1}, u_{h}^{P+1}\right)=0 .
$$

Then the inequality follows

$$
\left\|u_{h}^{P+1}\right\|_{\Omega}^{2}+\chi \lambda_{1}\left\|p_{h}^{P+1}\right\|_{\Omega}^{2} \leq\left\|u_{h}^{0}\right\|_{\Omega}^{2} .
$$

This finishes the proof of the stability result.

In order to simplify the analysis, the linear case $g(u)=u$ is studied.

Theorem 3.2 Suppose that $u\left(x, t_{n}\right)$ is the exact solution of problem (1.1), which is sufficiently smooth with bounded derivatives, and $u_{h}^{n}$ is the numerical solution of the fully discrete LDG scheme (3.5), it holds that

$$
\left\|u\left(x, t_{n}\right)-u_{h}^{n}\right\| \leq C\left(h^{k+1}+(\Delta t)^{2-\alpha}\right)
$$

where $C$ is a constant depending on $u, T, \alpha$.

Proof Consider the separation of numerical error in the form

$$
\begin{array}{lll}
e_{u}^{n}=u\left(x, t_{n}\right)-u_{h}^{n}=\xi_{u}^{n}-\eta_{u}^{n}, & \xi_{u}^{n}=\mathcal{P}^{-} e_{u}^{n}, & \eta_{u}^{n}=\mathcal{P}^{-} u\left(x, t_{n}\right)-u\left(x, t_{n}\right), \\
e_{p}^{n}=p\left(x, t_{n}\right)-p_{h}^{n}=\xi_{p}^{n}-\eta_{p}^{n}, & \xi_{p}^{n}=\mathcal{P} e_{p}^{n}, & \eta_{p}^{n}=\mathcal{P} p\left(x, t_{n}\right)-p\left(x, t_{n}\right) .
\end{array}
$$

Here $\eta_{u}^{n}$ and $\eta_{p}^{n}$ have been estimated by inequality (2.4). In what follows we are going to estimate $\xi_{u}^{n}$ and $\xi_{p}^{n}$. 
With flux (3.6), we can get the error equation

$$
\begin{aligned}
& \int_{\Omega} e_{u}^{n} v d x-\chi\left(\int_{\Omega} e_{u}^{n} v_{x} d x-\sum_{j=1}^{N}\left(\left(\left(e_{u}^{n}\right)^{-} v^{-}\right)_{j+\frac{1}{2}}-\left(\left(e_{u}^{n}\right)^{-} v^{+}\right)_{j-\frac{1}{2}}\right)\right) \\
& +\chi \lambda_{1}\left(\int_{\Omega} e_{p}^{n} v_{x} d x-\sum_{j=1}^{N}\left(\left(\left(e_{p}^{n}\right)^{+} v^{-}\right)_{j+\frac{1}{2}}-\left(\left(e_{p}^{n}\right)^{+} v^{+}\right)_{j-\frac{1}{2}}\right)\right) \\
& -\sum_{i=1}^{n-1}\left(s_{i-1}-s_{i}\right) \int_{\Omega} e_{u}^{n-i} v d x-s_{n-1} \int_{\Omega} e_{u}^{0} v d x \\
& +\chi \int_{\Omega} \gamma^{n}(x) v d x+\int_{\Omega} e_{p}^{n} w d x+\int_{\Omega} e_{u}^{n} w_{x} d x \\
& \quad-\sum_{j=1}^{N}\left(\left(\left(e_{u}^{n}\right)^{-} w^{-}\right)_{j+\frac{1}{2}}-\left(\left(e_{u}^{n}\right)^{-} w^{+}\right)_{j-\frac{1}{2}}\right)=0 .
\end{aligned}
$$

Using (3.19), error equation (3.20) can be written as follows:

$$
\begin{aligned}
& \int_{\Omega} \xi_{u}^{n} v d x-\chi\left(\int_{\Omega} \xi_{u}^{n} v_{x} d x-\sum_{j=1}^{N}\left(\left(\left(\xi_{u}^{n}\right)^{-} v^{-}\right)_{j+\frac{1}{2}}-\left(\left(\xi_{u}^{n}\right)^{-} v^{+}\right)_{j-\frac{1}{2}}\right)\right) \\
& +\chi \lambda_{1}\left(\int_{\Omega} \xi_{p}^{n} \nu_{x} d x-\sum_{j=1}^{N}\left(\left(\left(\xi_{p}^{n}\right)^{+} v^{-}\right)_{j+\frac{1}{2}}-\left(\left(\xi_{p}^{n}\right)^{+} v^{+}\right)_{j-\frac{1}{2}}\right)\right) \\
& +\int_{\Omega} \xi_{p}^{n} w d x+\int_{\Omega} \xi_{u}^{n} w_{x} d x-\sum_{j=1}^{N}\left(\left(\left(\xi_{u}^{n}\right)^{-} w^{-}\right)_{j+\frac{1}{2}}-\left(\left(\xi_{u}^{n}\right)^{-} w^{+}\right)_{j-\frac{1}{2}}\right) \\
& =\sum_{i=1}^{n-1}\left(s_{i-1}-s_{i}\right) \int_{\Omega} \xi_{u}^{n-i} v d x+s_{n-1} \int_{\Omega} \xi_{u}^{0} v d x-\chi \int_{\Omega} \gamma^{n}(x) v d x \\
& +\int_{\Omega}\left(\eta_{u}^{n}\right) v d x-\chi\left(\int_{\Omega}\left(\eta_{u}^{n}\right) v_{x} d x-\sum_{j=1}^{N}\left(\left(\left(\eta_{u}^{n}\right)^{-} v^{-}\right)_{j+\frac{1}{2}}-\left(\left(\eta_{u}^{n}\right)^{-} v^{+}\right)_{j-\frac{1}{2}}\right)\right) \\
& +\chi \lambda_{1}\left(\int_{\Omega}\left(\eta_{p}^{n}\right) v_{x} d x-\sum_{j=1}^{N}\left(\left(\left(\eta_{p}^{n}\right)^{+} v^{-}\right)_{j+\frac{1}{2}}-\left(\left(\eta_{p}^{n}\right)^{+} v^{+}\right)_{j-\frac{1}{2}}\right)\right) \\
& -\sum_{i=1}^{n-1}\left(s_{i-1}-s_{i}\right) \int_{\Omega}\left(\eta_{u}^{n-i}\right) v d x-s_{n-1} \int_{\Omega}\left(\eta_{u}^{0}\right) v d x+\int_{\Omega}\left(\eta_{p}^{n}\right) w d x+\int_{\Omega}\left(\eta_{u}^{n}\right) w_{x} d x \\
& -\sum_{j=1}^{N}\left(\left(\left(\eta_{u}^{n}\right)^{-} w^{-}\right)_{j+\frac{1}{2}}-\left(\left(\eta_{u}^{n}\right)^{-} w^{+}\right)_{j-\frac{1}{2}}\right)
\end{aligned}
$$

Taking the test functions $v=\xi_{u}^{n}, w=\chi \lambda_{1} \xi_{p}^{n}$ in (3.21), using properties (2.1) and (2.3), then the following equality holds:

$$
\begin{aligned}
& \int_{\Omega}\left(\xi_{u}^{n}\right)^{2} d x+\chi \lambda_{1} \int_{\Omega}\left(\xi_{p}^{n}\right)^{2} d x+\sum_{j=1}^{N} \frac{\chi \lambda_{1}}{2}\left[\xi_{u}^{n}\right]_{j-\frac{1}{2}}^{2} \\
& =\sum_{i=1}^{n-1}\left(s_{i-1}-s_{i}\right) \int_{\Omega} \xi_{u}^{n-i} \xi_{u}^{n} d x+s_{n-1} \int_{\Omega} \xi_{u}^{0} \xi_{u}^{n} d x-\chi \int_{\Omega} \gamma^{n}(x) \xi_{u}^{n} d x
\end{aligned}
$$


Yang Advances in Difference Equations

(2020)

2020:182

Page 8 of 11

$$
\begin{aligned}
& +\int_{\Omega}\left(\eta_{u}^{n}\right) \xi_{u}^{n} d x-\chi\left(\int_{\Omega}\left(\eta_{u}^{n}\right)\left(\xi_{u}^{n}\right)_{x} d x-\sum_{j=1}^{N}\left(\left(\left(\eta_{u}^{n}\right)^{-}\left(\xi_{u}^{n}\right)^{-}\right)_{j+\frac{1}{2}}-\left(\left(\eta_{u}^{n}\right)^{-}\left(\xi_{u}^{n}\right)^{+}\right)_{j-\frac{1}{2}}\right)\right) \\
& +\chi \lambda_{1}\left(\int_{\Omega}\left(\eta_{p}^{n}\right)\left(\xi_{u}^{n}\right)_{x} d x-\sum_{j=1}^{N}\left(\left(\left(\eta_{p}^{n}\right)^{+}\left(\xi_{u}^{n}\right)^{-}\right)_{j+\frac{1}{2}}-\left(\left(\eta_{p}^{n}\right)^{+}\left(\xi_{u}^{n}\right)^{+}\right)_{j-\frac{1}{2}}\right)\right) \\
& +\sum_{i=1}^{n-1}\left(s_{i-1}-s_{i}\right) \int_{\Omega}\left(\eta_{u}^{n-i}\right) \xi_{u}^{n} d x-s_{n-1} \int_{\Omega}\left(\eta_{u}^{0}\right) \xi_{u}^{n} d x+\int_{\Omega}\left(\eta_{p}^{n}\right)\left(\chi \lambda_{1} \xi_{p}^{n}\right) d x \\
& +\int_{\Omega}\left(\eta_{u}^{n}\right)\left(\chi \lambda_{1} \xi_{p}^{n}\right)_{x} d x-\sum_{j=1}^{N}\left(\left(\left(\eta_{u}^{n}\right)^{-}\left(\chi \lambda_{1} \xi_{p}^{n}\right)^{-}\right)_{j+\frac{1}{2}}-\left(\left(\eta_{u}^{n}\right)^{-}\left(\chi \lambda_{1} \xi_{p}^{n}\right)^{+}\right)_{j-\frac{1}{2}}\right) \\
& =\sum_{i=1}^{n-1}\left(s_{i-1}-s_{i}\right) \int_{\Omega} \xi_{u}^{n-i} \xi_{u}^{n} d x+s_{n-1} \int_{\Omega} \xi_{u}^{0} \xi_{u}^{n} d x-\chi \int_{\Omega} \gamma^{n}(x) \xi_{u}^{n} d x \\
& +\int_{\Omega}\left(\eta_{u}^{n}\right) \xi_{u}^{n} d x+\chi \lambda_{1} \sum_{j=1}^{N}\left(\left(\eta_{p}^{n}\right)^{+}\left[\xi_{u}^{n}\right]\right)_{j-\frac{1}{2}} \\
& +\sum_{i=1}^{n-1}\left(s_{i-1}-s_{i}\right) \int_{\Omega}\left(\eta_{u}^{n-i}\right) \xi_{u}^{n} d x-s_{n-1} \int_{\Omega}\left(\eta_{u}^{0}\right) \xi_{u}^{n} d x .
\end{aligned}
$$

Error estimates (3.18) will be proved by mathematical induction. First, we consider the case $n=1$, that is,

$$
\begin{aligned}
& \int_{\Omega}\left(\xi_{u}^{1}\right)^{2} d x+\chi \lambda_{1} \int_{\Omega}\left(\xi_{p}^{1}\right)^{2} d x+\sum_{j=1}^{N} \frac{\chi}{2}\left[\xi_{u}^{1}\right]_{j-\frac{1}{2}}^{2} \\
& =\int_{\Omega} \xi_{u}^{0} \xi_{u}^{1} d x-\chi \int_{\Omega} \gamma^{1}(x) \xi_{u}^{1} d x+\int_{\Omega}\left(\eta_{u}^{1}\right) \xi_{u}^{1} d x \\
& \quad+\chi \lambda_{1} \sum_{j=1}^{N}\left(\left(\eta_{p}^{1}\right)^{+}\left[\xi_{u}^{1}\right]\right)_{j-\frac{1}{2}}-\int_{\Omega}\left(\eta_{u}^{0}\right) \xi_{u}^{1} d x .
\end{aligned}
$$

We know

$$
\left\|\xi_{u}^{0}\right\| \leq C h^{k+1}, \quad a b \leq \varepsilon a^{2}+\frac{1}{4 \varepsilon} b^{2}
$$

and based on (2.4), we have

$$
\begin{gathered}
\int_{\Omega}\left(\xi_{u}^{1}\right)^{2} d x+\chi \lambda_{1} \int_{\Omega}\left(\xi_{p}^{1}\right)^{2} d x+\sum_{j=1}^{N} \frac{\chi}{2}\left[\xi_{u}^{1}\right]_{j-\frac{1}{2}}^{2} \\
\leq\left(\left\|\xi_{u}^{0}\right\|+\chi\left\|\gamma^{1}(x)\right\|+\left\|\left(\eta_{u}^{1}\right)\right\|+\left\|\left(\eta_{u}^{0}\right)\right\|\right)\left\|\xi_{u}^{1}\right\| \\
\quad+\frac{\chi \lambda_{1}}{4} \sum_{j=1}^{N}\left(\left(\eta_{p}^{1}\right)^{+}\right)_{j-\frac{1}{2}}^{2}+\varepsilon \chi \lambda_{1} \sum_{j=1}^{N}\left(\left[\xi_{u}^{1}\right]\right)_{j-\frac{1}{2}} \\
\leq C\left(h^{k+1}+(\Delta t)^{2-\alpha}\right)^{2}+\varepsilon\left\|\xi_{u}^{1}\right\|^{2}+\varepsilon \chi \lambda_{2} \sum_{j=1}^{N}\left(\left[\xi_{u}^{1}\right]\right)_{j-\frac{1}{2}} .
\end{gathered}
$$


Choosing small $\varepsilon$, we know

$$
\left\|\xi_{u}^{1}\right\|_{\Omega} \leq C\left(h^{k+1}+(\Delta t)^{2-\alpha}\right) .
$$

Then suppose that

$$
\left\|\xi_{u}^{m}\right\|_{\Omega} \leq C\left(h^{k+1}+(\Delta t)^{2-\alpha}\right), \quad m=1,2, \ldots, K
$$

Let $n=K+1$ in (3.22), we have

$$
\begin{aligned}
\int_{\Omega}\left(\xi_{u}^{K+1}\right)^{2} d x+\chi \lambda_{1} \int_{\Omega}\left(\xi_{p}^{K+1}\right)^{2} d x+\sum_{j=1}^{N} \frac{\chi}{2}\left[\xi_{u}^{K+1}\right]_{j-\frac{1}{2}}^{2} \\
=\sum_{i=1}^{K}\left(s_{i-1}-s_{i}\right) \int_{\Omega} \xi_{u}^{K+1-i} \xi_{u}^{K+1} d x+s_{K} \int_{\Omega} \xi_{u}^{0} \xi_{u}^{K+1} d x \\
\quad-\chi \int_{\Omega} \gamma^{K+1}(x) \xi_{u}^{K+1} d x+\int_{\Omega}\left(\eta_{u}^{K+1}\right) \xi_{u}^{K+1} d x+\chi \lambda_{1} \sum_{j=1}^{N}\left(\left(\eta_{p}^{K+1}\right)^{+}\left[\xi_{u}^{K+1}\right]\right)_{j-\frac{1}{2}} \\
\quad-\sum_{i=1}^{K}\left(s_{i-1}-s_{i}\right) \int_{\Omega}\left(\eta_{u}^{K+1-i}\right) \xi_{u}^{K+1} d x-s_{K} \int_{\Omega}\left(\eta_{u}^{0}\right) \xi_{u}^{K+1} d x \\
\leq C\left(h^{k+1}+(\Delta t)^{2-\alpha}\right)^{2}+\varepsilon\left\|\xi_{u}^{K+1}\right\|^{2}+\varepsilon \chi \lambda_{1} \sum_{j=1}^{N}\left(\left[\xi_{u}^{K+1}\right]\right)_{j-\frac{1}{2}} .
\end{aligned}
$$

We can obtain

$$
\left\|\xi_{u}^{K+1}\right\| \leq C\left(h^{k+1}+(\Delta t)^{2-\alpha}\right) .
$$

By using the triangle inequality and the interpolating property (2.4), we have Theorem 3.2.

\section{Numerical examples}

In this section, we present some numerical experiments for the proposed local discontinuous Galerkin method to illustrate its capability.

Example 4.1 Consider problem (1.1) when $\lambda_{1}=1$ in $\Omega=[0,2]$, the forcing term

$$
f(x, t)=\left(\frac{24 t^{4-\alpha}}{\Gamma(5-\alpha)}+\pi^{2}\left(t^{4}+1\right)\right) \sin (\pi x)+\pi \sin (\pi x) \cos (\pi x)\left(t^{4}+1\right)^{2}
$$

and the exact solution is $u(x, t)=\left(t^{4}+1\right) \sin (\pi x)$. The $L^{2}$ and $L^{\infty}$ errors and the numerical orders of accuracy at time $T=1$ for several $\alpha$ are contained in Tables $1-2$. We can see that the convergence rates are well displayed and justify our theoretical results.

\section{Conclusion}

In this paper we have presented a fully discrete local discontinuous Galerkin method for solving a class of fractional Burgers-type equations. Numerical experiments show that 
Table 1 Numerical accuracy of scheme (3.5) with $M=1000, \alpha=0.3$

\begin{tabular}{rrllll}
\hline & $N$ & $L^{2}$-error & Order & $L^{\infty}$-error & Order \\
\hline$p^{0}$ & 5 & $4.7435116474654447 \mathrm{e}-01$ & - & $5.230485703724679 \mathrm{e}-01$ & - \\
& 10 & $2.453322326385340 \mathrm{e}-01$ & 0.95 & $2.745599439848157 \mathrm{e}-01$ & 0.92 \\
& 15 & $1.653406665596814 \mathrm{e}-01$ & 0.97 & $1.860855920209763 \mathrm{e}-01$ & 0.95 \\
& 20 & $1.242678198594019 \mathrm{e}-01$ & 0.99 & $1.415701279740494 \mathrm{e}-01$ & 0.95 \\
$P^{1}$ & 5 & $7.2565175368555258 \mathrm{e}-02$ & - & $9.635412347824566 \mathrm{e}-02$ & - \\
& 10 & $1.873884491779974 \mathrm{e}-02$ & 1.95 & $2.552822346426505 \mathrm{e}-02$ & 1.91 \\
& 15 & $8.4322948346669999 \mathrm{e}-03$ & 1.97 & $1.166039812718010 \mathrm{e}-02$ & 1.93 \\
& 20 & $4.766101927280031 \mathrm{e}-03$ & 1.98 & $6.630816791277043 \mathrm{e}-03$ & 1.96 \\
$P^{2}$ & 5 & $1.097532348354286 \mathrm{e}-02$ & - & $2.164416564453414 \mathrm{e}-02$ & - \\
& 10 & $1.477316800540677 \mathrm{e}-03$ & 2.89 & $2.900434572823438 \mathrm{e}-03$ & 2.90 \\
& 15 & $4.535585735151620 \mathrm{e}-04$ & 2.91 & $8.863974464363477 \mathrm{e}-04$ & 2.92 \\
& $1.956222641594544 \mathrm{e}-04$ & 2.92 & $3.793487019026590 \mathrm{e}-04$ & 2.95 \\
\hline & & & & &
\end{tabular}

Table 2 Numerical accuracy of scheme (3.5) with $M=1000, \alpha=0.7$

\begin{tabular}{|c|c|c|c|c|c|}
\hline & $\mathrm{N}$ & $L^{2}$-error & Order & $L^{\infty}$-error & Order \\
\hline$p^{0}$ & $\begin{array}{r}5 \\
10 \\
15 \\
20\end{array}$ & $\begin{array}{l}4.348211568435824 \mathrm{e}-01 \\
2.424520324538541 \mathrm{e}-01 \\
1.352400215461305 \mathrm{e}-01 \\
1.121874628534372 \mathrm{e}-01\end{array}$ & $\begin{array}{l}- \\
0.95 \\
0.97 \\
0.99\end{array}$ & $\begin{array}{l}5.042185301727834 \mathrm{e}-01 \\
2.624532134506741 \mathrm{e}-01 \\
1.589644242186741 \mathrm{e}-01 \\
1.289403452797642 \mathrm{e}-01\end{array}$ & $\begin{array}{l}- \\
0.92 \\
0.95 \\
0.95\end{array}$ \\
\hline$P^{1}$ & $\begin{array}{r}5 \\
10 \\
15 \\
20\end{array}$ & $\begin{array}{l}7.684216542820121 \mathrm{e}-02 \\
1.687534622310582 \mathrm{e}-02 \\
8.534804436315641 \mathrm{e}-03 \\
4.521403201237941 \mathrm{e}-03\end{array}$ & $\begin{array}{l}- \\
1.95 \\
1.97 \\
1.98\end{array}$ & $\begin{array}{l}9.154712784824911 \mathrm{e}-02 \\
2.164722782426461 \mathrm{e}-02 \\
1.247106712856485 \mathrm{e}-02 \\
6.503791571074942 \mathrm{e}-03\end{array}$ & $\begin{array}{l}- \\
1.91 \\
1.93 \\
1.96\end{array}$ \\
\hline$p^{2}$ & $\begin{array}{r}5 \\
10 \\
15 \\
20\end{array}$ & $\begin{array}{l}1.164727942789012 \mathrm{e}-02 \\
1.348554214954641 \mathrm{e}-03 \\
4.054195123465723 \mathrm{e}-04 \\
1.264756425642313 \mathrm{e}-04\end{array}$ & $\begin{array}{l}- \\
2.89 \\
2.91 \\
2.92\end{array}$ & $\begin{array}{l}2.237484026942101 \mathrm{e}-02 \\
2.899243648502348 \mathrm{e}-03 \\
8.057494485268102 \mathrm{e}-04 \\
3.752416915626942 \mathrm{e}-04\end{array}$ & $\begin{array}{l}- \\
2.90 \\
2.92 \\
2.95\end{array}$ \\
\hline
\end{tabular}

our scheme is very effective. The advantages of the method are its flexibility in terms of mesh and shape functions, and it can achieve a high order of convergence. In future we would study a local discontinuous Galerkin method and an alternating direction implicit/iterative scheme to solve the two- or higher-dimensional problems.

Acknowledgements

The authors would like to express their sincere appreciation to the editor and reviewers for their helpful and detailed comments in improving the presentation and quality of the paper.

Funding

This work is supported by the Foundation of He'nan Educational Committee (19A110005).

Availability of data and materials

Data sharing not applicable to this article as no datasets were generated or analysed during the current study.

\section{Competing interests}

The author declares that they have no competing interests.

Authors' contributions

All authors contributed equally to the writing of this paper. All authors read and approved the final manuscript.

\section{Publisher's Note}

Springer Nature remains neutral with regard to jurisdictional claims in published maps and institutional affiliations.

Received: 28 August 2019 Accepted: 6 March 2020 Published online: 28 April 2020

References

1. Burgers, J.: A Mathematical Model Illustrating the Theory of Turbulence. Academic Press, New York (1948) 
2. Ciarlet, P.: The Finite Element Method for Elliptic Problems. North-Holland, Amsterdam (1978)

3. El-Danaf, T., Hadhoud, A.: Parametric spline functions for the solution of the one time fractional Burgers' equation. Appl. Math. Model. 36, 4557-4564 (2012)

4. Gu, X., Huang, T., Ji, C., Carpentieri, B., Alikhanov, A.: Fast iterative method with a second-order implicit difference scheme for time-space fractional convection-diffusion equation. J. Sci. Comput. 72, 957-985 (2017)

5. Inc, M.: The approximate and exact solutions of the space- and time-fractional Burgers equation with initial conditions by variational iteration method. J. Math. Anal. Appl. 345, 476-484 (2008)

6. Jiang, Y., Ma, J.: High-order finite element methods for time-fractional partial differential equations. J. Comput. Appl. Math. 235, 3285-3290 (2011)

7. Li, M., Gu, X., Huang, C., Fei, M., Zhang, G.: A fast linearized conservative finite element method for the strongly coupled nonlinear fractional Schrödinger equations. J. Comput. Phys. 358, 256-282 (2018)

8. Meerschaert, M.M., Nane, E., Vellaisamy, P.: Distributed-order fractional diffusions on bounded domains. J. Math. Anal. Appl. 379, 216-228 (2011)

9. Morgado, M.L., Rebelo, M.: Numerical approximation of distributed order reaction-diffusion equations. J. Comput. Appl. Math. 275, 216-227 (2015)

10. Naber, M.: Distributed order fractional sub-diffusion. Fractals 12, 23-32 (2004)

11. Podlubny, I.: Fractional Differential Equations. Academic Press, San Diego (1999)

12. Ren, Z., Wei, L., He, Y., Wang, S.: Numerical analysis of an implicit fully discrete local discontinuous Galerkin method for the fractional Zakharov-Kuznetsov equation. Math. Model. Anal. 17, 558-570 (2012)

13. Vong, S., Lyu, P.: Unconditional convergence in maximum-norm of a second-order linearized scheme for a time-fractional Burgers-type equation. J. Sci. Comput. 76, 1252-1273 (2018)

14. Wang, Q.: Numerical solutions for fractional KdV-Burgers equation by Adomian decomposition method. Appl. Math. Comput. 182, 1048-1055 (2006)

15. Wei, L.L.: Analysis of a new finite difference/local discontinuous Galerkin method for the fractional diffusion-wave equation. Appl. Math. Comput. 304, 180-189 (2017)

16. Wei, L.L.: Stability and convergence of a fully discrete local discontinuous Galerkin method for multi-term time fractional diffusion equations. Numer. Algorithms 76, 695-707 (2017)

17. Wei, L.L.: Analysis of a new finite difference/local discontinuous Galerkin method for the fractional Cattaneo equation. Numer. Algorithms 77, 675-690 (2018)

18. Wei, L.L., He, Y.N.: Analysis of a fully discrete local discontinuous Galerkin method for time-fractional fourth-order problems. Appl. Math. Model. 38, 1511-1522 (2014)

19. Wei, L.L., He, Y.N., Tang, B.: Analysis of the fractional Kawahara equation using an implicit fully discrete local discontinuous Galerkin method. Numer. Methods Partial Differ. Equ. 29, 1441-1458 (2013)

20. Zhang, Q., Shu, C.-W.: Error estimate for the third order explicit Runge-Kutta discontinuous Galerkin method for a linear hyperbolic equation with discontinuous initial solution. Numer. Math. 126, 703-740 (2014)

21. Zhang, X., Tang, B., He, Y.: Homotopy analysis method for higher-order fractional integro-differential equations. Comput. Math. Appl. 62, 3194-3203 (2011)

\section{Submit your manuscript to a SpringerOpen ${ }^{\circ}$ journal and benefit from:}

- Convenient online submission

- Rigorous peer review

- Open access: articles freely available online

- High visibility within the field

- Retaining the copyright to your article

Submit your next manuscript at $\boldsymbol{s p r i n g e r o p e n . c o m ~}$ 